
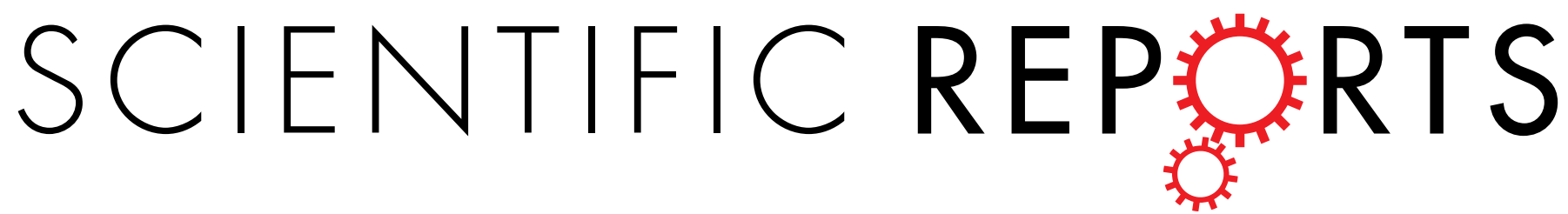

\title{
OPEN
}

\section{Double knockout of Bax and Bak from kidney proximal tubules reduces unilateral urethral}

Received: 31 October 2016

Accepted: 15 February 2017

Published: 20 March 2017

\section{obstruction associated apoptosis and renal interstitial fibrosis}

\author{
Shuqin $\mathrm{Mei}^{1,2}$, Lin $\mathrm{Li}^{1,2}$, Qingqing Wei ${ }^{2}$, Jielu $\mathrm{Hao}^{1,2}$, Yunchao $\mathrm{Su}^{3}$, Changlin $\mathrm{Mei}^{1}$ \& \\ Zheng Dong ${ }^{2,4}$
}

Interstitial fibrosis, a common pathological feature of chronic kidney diseases, is often associated with apoptosis in renal tissues. To determine the associated apoptotic pathway and its role in renal interstitial fibrosis, we established a mouse model in which Bax and Bak, two critical genes in the intrinsic pathway of apoptosis, were deleted specifically from kidney proximal tubules and used this model to examine renal apoptosis and interstitial fibrosis following unilateral urethral obstruction (UUO). It was shown that double knockout of Bax and Bak from proximal tubules attenuated renal tubular cell apoptosis and suppressed renal interstitial fibrosis in UUO. The results indicate that the intrinsic pathway of apoptosis contributes significantly to the tubular apoptosis and renal interstitial fibrosis in kidney diseases.

Renal interstitial fibrosis is a common pathological feature during the progression of kidney disease to end stage renal failure ${ }^{1,2}$. While characterized by interstitial myofibroblast activation and expansion and massive extracellular matrix protein accumulation ${ }^{3}$, renal interstitial fibrosis is often accompanied by renal cell injury and death and inflammation. In fact, both renal cell death and inflammation have been suggested to contribute to the development of renal interstitial fibrosis. However, it is not fully understood whether renal tubular apoptosis is a key factor in interstitial fibrosis and, if it is, what apoptotic pathway is mainly responsible.

There are two major pathways of apoptosis ${ }^{4}$. The extrinsic pathway is initiated by the binding of death receptors, e.g. Fas and TNForeceptors, by death ligands, leading to the activation of caspase- 8 and downstream executioner caspases. In contrast, the intrinsic pathway is centered on mitochondria and thus is frequently referred to as mitochondrial pathway of apoptosis. The intrinsic pathway is characterized by the permeabilization of mitochondrial outer membrane causing the release of apoptogenic factors, especially cytochrome $c$, which induce the activation of caspase- 9 and down-stream caspases ${ }^{5,6}$. Bax and Bak are the key proapoptotic Bcl-2 family proteins that are activated in the intrinsic pathway to permeabilize mitochondrial outer membrane for apoptosis ${ }^{7}$. Our previous work has established a critical role for Bax and Bak in renal cell apoptosis in experimental models of acute kidney injury induced by ischemia/reperfusion and cisplatin nephrotoxicity ${ }^{5}$.

In the present study, we have produced renal tubule-specific Bax/Bak double knockout mouse model and tested the effect of Bax/Bak double knockout on tubular apoptosis and renal interstitial fibrosis in the model of unilateral urethral obstruction (UUO).

${ }^{1}$ Kidney Institute and Department of Nephrology, Shanghai Changzheng Hospital, Second Military Medical University, Shanghai, China. ${ }^{2}$ Department of Cellular Biology and Anatomy, Medical College of Georgia at Augusta University and Charlie Norwood VA medical Center, Augusta, Georgia, USA. ${ }^{3}$ Department of Pharmacology and Toxicology, Medical College of Georgia at Augusta University and Charlie Norwood VA medical Center, Augusta, Georgia, USA. 'Department of Nephrology, The Second Xiangya Hospital of Central South University, Changsha, China. Correspondence and requests for materials should be addressed to C.M. (email: chlmei@126.com) or Z.D. (email: zdong@augusta.edu) 
A

\begin{tabular}{|c|c|c|}
\hline Male & & Female \\
\hline Bax (WTMT)Bak(flox/flox)XY & $\mathbf{x}$ & Bax(flox/flox)Bak(WT/WT) $X^{\text {Cre }} \mathrm{X}$ \\
\hline Bax (flox/WT)Bak(flox/WT)XY & $\mathbf{x}$ & $\operatorname{Bax}\left(\right.$ flox/WT)Bak(flox/WT)X $\mathrm{X}^{\text {cre } X}$ \\
\hline Bax (flox/flox)Bak(flox/flox)XY & $x$ & Bax(flox/flox)Bak(flox/flox) $X^{\text {Cre } X}$ \\
\hline
\end{tabular}

B

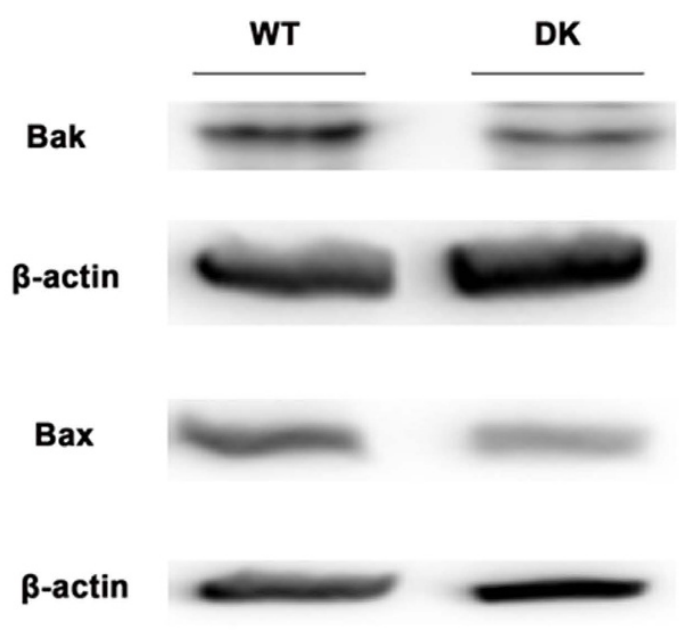

Figure 1. Establishment of proximal tubule-specific Bax and Bak-double knockout (PT-Bax/Bak-DK) mouse model. (A) Schematics showing the breeding protocol for generating PT-Bax/Bak-DK mice by crossing $\operatorname{Bax}\left(\right.$ flox/flox)Bak(WT/WT) $\mathrm{X}^{\mathrm{CRE} X}$ mice with Bax(WT/WT)Bak(flox/flox)XY mice. (B) Immunoblot analysis of renal cortical tissues to confirm the decreases in Bax and Bak expression in PT-Bax/Bak-DK mice.

\section{Results}

In our previously work, we demonstrated that ischemic acute kidney injury was ameliorated in renal proximal tubule Bax knockout mice [PT-Bax $(-/-)]$ mice and global Bak knockout $[\mathrm{Bak}(-/-)]$ mice, supporting a critical role of Bax/Bak-mediated mitochondrial pathway of apoptosis ${ }^{5}$. But the role of Bax and Bak in chronic kidney pathology, such as that in UUO, was not entirely clear. To address this, we first generated Bax(flox/flox) Bak(WT/WT) X ${ }^{\mathrm{CRE}} \mathrm{X}$ mice by breeding Bax(flox/flox)Bak(WT/WT)XY and Bax(WT/WT)Bak(WT/WT) $\mathrm{X}^{\mathrm{CRE}} \mathrm{X}^{\mathrm{CRE}}$ (PEPCK-CRE) mice. We then back-crossed Bax(flox/flox)Bak(WT/WT) $\mathrm{X}^{\mathrm{CRE}} \mathrm{X}$ mice with Bax(WT/ WT)Bak(flox/flox)XY mice for 2 generations to produce proximal tubule-specific Bax/Bak double knockout (PT-Bax/Bak-DK) mice (Fig. 1A). In addition to genotyping results (not shown), Bax and Bak depletion in proximal tubular cells in PT-Bax/Bak-DK mice was confirmed by Western blot analysis (Fig. 1B). Of note, there were residual Bax and Bak signals in the renal cortical tissues in PT-Bax/Bak-DK mice, because the knockout was restricted in proximal tubules and the expression of Bax and Bak in other cell types was not affected.

Double knockout of tubular Bax and Bak inhibits UUO-induced renal apoptosis. UUO is a commonly used model for kidney fibrosis research. The pathological changes in UUO include tubular cell injury and death, phenotypic alterations of renal cells, and interstitial inflammation ${ }^{8}$. By TUNEL staining, we detected less apoptotic cells in PT-Bax/Bak-DK kidney tissues than WT kidney tissues after UUO 4, 7, and 14 days (Fig. 2A and B), suggest a role of tubular Bax and Bak in apoptosis in UUO.

Double knockout of tubular Bax and Bak alleviates UUO-associated kidney fibrosis. In order to elucidate the effect of Bax and Bak gene knockout in renal interstitial fibrosis, we detected collagen deposition by Masson's trichrome staining and the expression of fibrotic hallmark proteins ${ }^{9,10}$. As shown by Fig. $3 \mathrm{~A}$, there was a time-dependent increase in blue Masson's trichrome staining during UUO. Importantly, the wild type tissues clearly displayed higher Masson's trichrome staining than PT-Bax/Bak-DK kidney tissues in renal interstitial area at 7 and 14 days of UUO, but no notable difference at 4 days of UUO (Fig. 3A and B). Consistently, the level of 
A

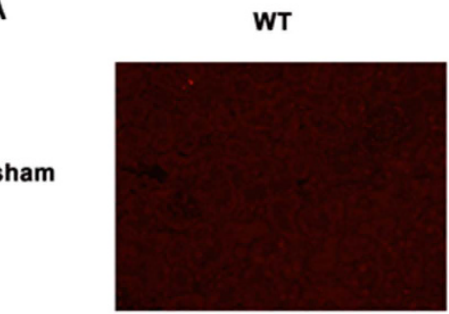

UUO 4D

UUO 7D

UUO 14D
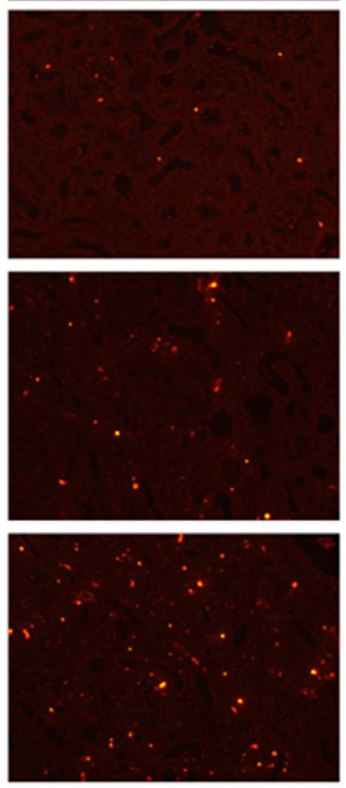

B

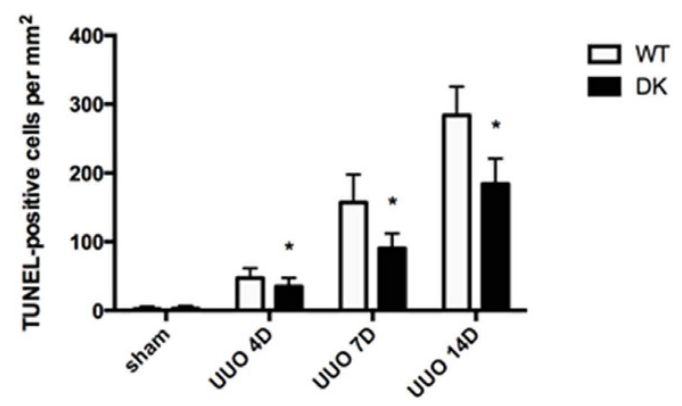

DK
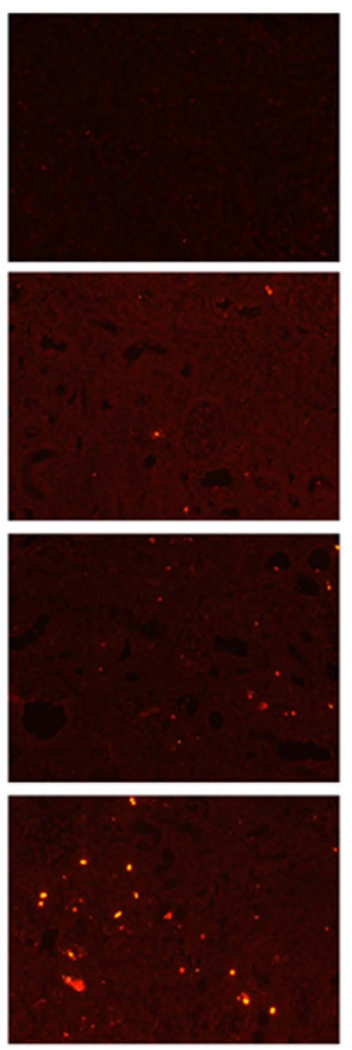
WT k

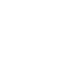


A WT DK

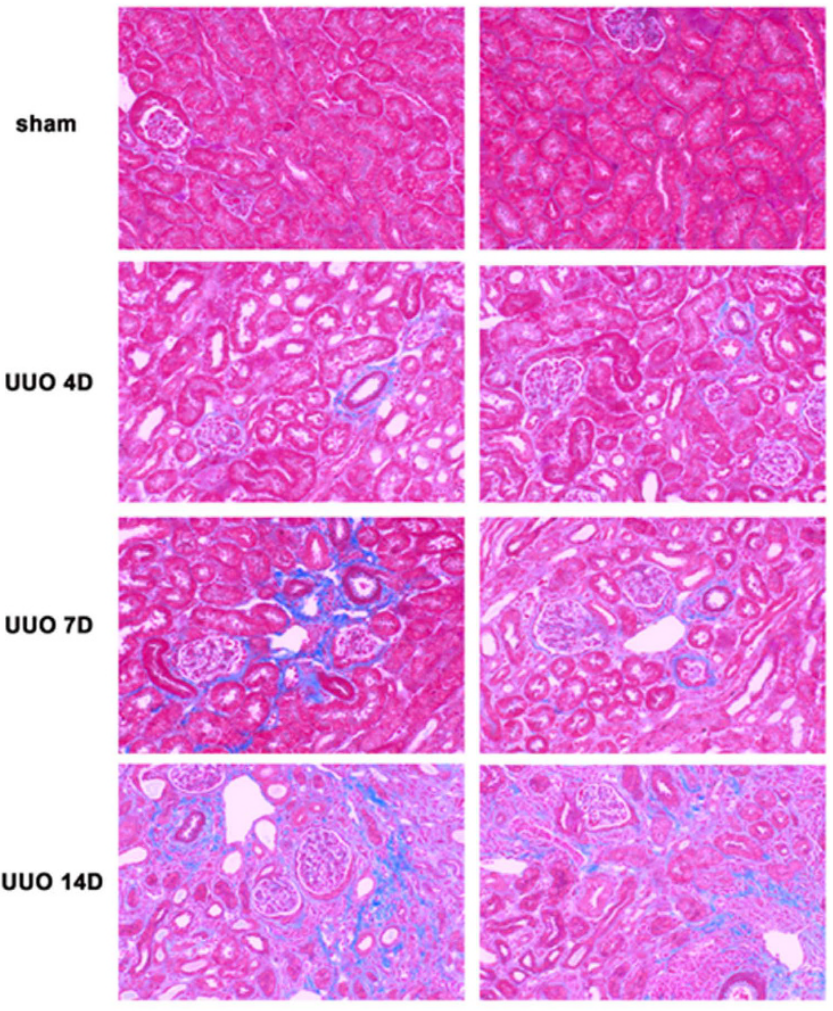

B

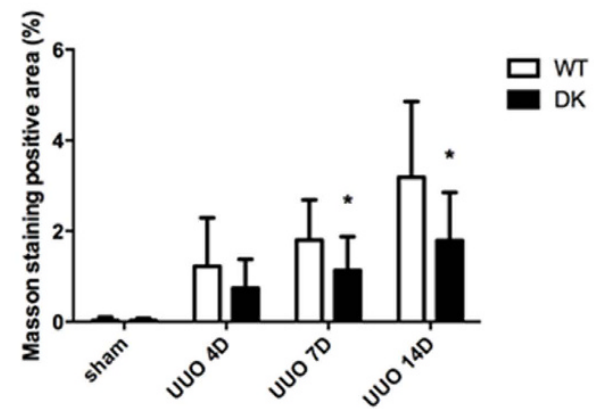

Figure 3. UUO-associated collagen deposition in kidney tissues is reduced in PT-Bax/Bak-DK mice. PTBax/Bak-DK and littermate WT mice (10-12 weeks old) were subjected to UUO or sham surgery, and then sacrificed to harvest the kidneys at 4, 7 and 14 days for paraffin-embedding and Masson's trichrome staining. (A) Representative images of Masson's trichrome staining. (B) Quantification of Masson's trichrome positive areas in whole kidney tissue fields. Data were expressed as mean \pm SD. ${ }^{\star} P<0.05$, significant difference between WT and PT-Bax/Bak-DK groups.

Bak, stress-related apoptosis is diminished, supporting a critical role of Bax and Bak in the extrinsic pathway of apoptosis $^{17,18}$. In kidneys, our previous work showed Bax activation in cisplatin-induced acute kidney injury and demonstrated that germline knockout of Bax could reduce cisplatin-induced renal apoptosis in mice ${ }^{19}$. Moreover, we demonstrated a critical role of Bax and Bak in ischemic AKI by using global and proximal tubule-specific knockout mouse model ${ }^{5}$. In that study, we noticed that global knockout of Bax did not protect againt ischemic AKI because of the enhanced neutrophil infiltration, but proximal tubule specific knockout of Bax could ameliorate ischemic AKI. In contrast, global Bak knockout mice model displayed protective effects from ischemic AKI by preserve mitochondrial dynamics and integrity ${ }^{5}$. It is noteworthy that Bax and Bak knockout mice attenuated apoptosis in ischemic AKI without affecting tubular necrosis ${ }^{5}$, suggesting a specific role of Bax and Bak in the regulation of apoptosis in kidneys. Despite these studies, the role of Bax, Bak and the intrinsic pathway of apoptosis in chronic conditions of kidney diseases remains less clear. In this study, we established a mouse model of double knockout of Bax and Bak from renal proximal tubules and used this model to demonstrate the role of the intrinsic pathway of apoptosis in UUO-associated apoptosis and interstitial fibrosis.

What role does tubular apoptosis play in fibrosis and renal pathologies in CKD or chronic kidney disease? Johnson and Dipietro showed that apoptosis had roles in the initiation and propagation of organ fibrosis via 
A
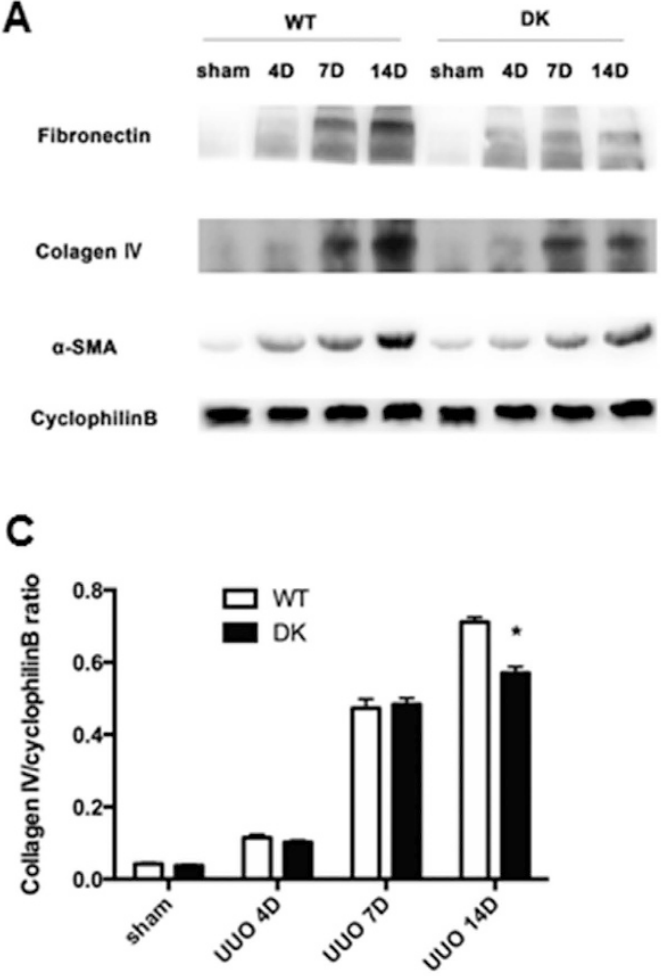

B
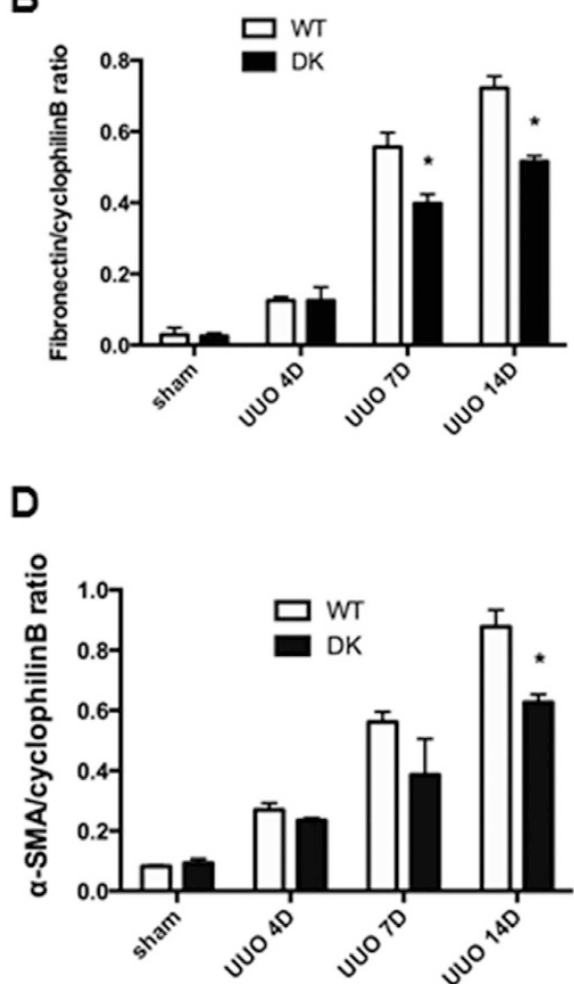

Figure 4. UUO-associated expression of fibrosis and extracellular matrix markers in kidney tissues is suppressed in PT-Bax/Bak-DK mice. PT-Bax/Bak-DK and littermate WT mice (10-12 weeks old) were subjected to UUO or sham surgery, and then sacrificed to harvest the kidneys at 4,7 and 14 days. (A) Immunoblot analysis of kidney tissues for fibronectin, $\alpha$-SMA, collagen IV and cyclophilin B (loading control). (B) Densitometry analysis of the immunoblot signals of fibronectin, $\alpha$-SMA, and collagen IV as a ratio to cyclophilinB. Data were expressed as mean $\pm \mathrm{SD} .{ }^{*} P<0.05$, significant difference between WT and PT-Bax/ Bak-DK groups.

directly or indirectly pathways ${ }^{20}$. Indirectly, apoptosis may elicit inflammation to stimulate fibrosis. In this regard, upon activation macrophages may secrete profibrotic cytokines and growth factors, such as tumor necrosis factor $\alpha(\mathrm{TNF} \alpha)$, interleukin (IL)-6, IL-10 and transforming growth factor $\beta 1$ (TGF- $\beta 1)^{21,22}$. On the other hand, apoptotic cells may directly affect the fibroblast cells and myofibroblasts to enhance their proliferation and activation into profibrotic phenotypes ${ }^{3,23,24}$. In our study, we detected apoptotic cells in wild-type kidney tissues mostly in the outer strip of out medulla beginning at UUO 4 day and increasing gradually after UUO 7 days and 14 days. Compared to wild-type, PT-Bax/Bak-DK tissues showed less apoptotic cells in each time point after UUO. Along with apoptosis, there was a continuous increase of renal fibrosis in wild-type tissues, which was also suppressed in PT-Bax/Bak-DK mice, indicating a correlation of tubular cell apoptosis and interstitial fibrosis (Fig. 3A and B). These results, together with a recent study ${ }^{25}$, support a role of tubular cell apoptosis in fibrogenesis in the intersitium. Of note, in our study there was residual apoptosis in PT-Bax/Bak-DK mice during UUO (Fig. 2). One possibility is that in addition to Bax/Bak-mediated mitochondrial pathway, other pathways of apoptosis, e.g. death receptor-mediated extrinsic pathway, may also contribute to tubular cell apoptosis in UUO. Alternatively, in the PT-Bax/Bak-DK model, Bax and Bak ablation was expected to occur in $70-80 \%$ proximal tubule cells due to the efficacy of PEPCK-Cre expression ${ }^{26}$, and the remaining 20-30\% cells still contained Bax and Bak for apoptosis. Finally, some of the residual apoptosis might occur in other cell types such as distal tubules.

In conclusion, this study has established a renal proximal tubule Bax/Bak double knockout mouse model. By using this model, we have demonstrated a role of Bax/Bak-mediated intrinsic pathway in renal apoptosis during UUO. We further suggest that renal tubular apoptosis contributes to interstitial fibrosis.

\section{Materials and Methods}

Animals. The mouse line with floxed Bax gene was originally obtained from Dr. Stanley Korsmeyer at DanaFarber Cancer Institute (Boston, MA) ${ }^{27}$. The mouse with floxed Bak gene were generated in our laboratory by a standard transgenic procedure. Both mouse lines have a mixed 129 and C57BL/6 background. PEPCK-CRE mouse line was kindly provided by Dr. Volker Haase at Vanderbilt University school of Medicine (Nashville, $\mathrm{TN})^{26}$. These mice were crossed for several generations as depicted in Fig. 1A to produce renal proximal tubuleBax/Bak double knockout mice (PT-Bax/Bak-DK) and wild type mice (WT). The genotypes of the mice were verified by PCR-based genotyping. Male animals aged 10-12 weeks were used in this study. All animals were maintained at Charlie Norwood VA Medical Center under 12-hour light/12-hour dark pattern with free access to 
food and water. All experiments were carried out according to the protocol approved by the Institute Animal Care and Usage Committee in Charlie Norwood VA Medical Center, Augusta, GA.

Regents. The sources of primary antibodies were as follows: Fibronectin, $\alpha$-SMA, Collagen I and Collagen IV were purchased from Abcam (Cambridge, MA), and $\beta$-actin was from Sigma (St Louis, MO). All second antibodies were purchased from Jackson Immuno Research Laboratories Inc (West Grove, PA). Paraformaldehyde was from Sigma (St. Louis, MO).

Animal surgery. PT-Bax/Bak-DK and WT mice were anesthetized by intraperitoneal injection with $60 \mathrm{mg} /$ $\mathrm{kg}$ pentobarbital. To relieve distress, $0.05 \mathrm{mg} / \mathrm{kg}$ buprenex was given after surgery. During surgery, mice were kept on a temperature-controlled operating table to maintain body temperature. The left ureter was dissected out and ligated by 4.0 silk at two points along the length, while the sham group underwent the same procedure except for ureter ligation. After 4, 7, 14 days of UUO, mice were sacrificed and kidney were harvested for pathological and immunoblotting analyses.

Immunoblot analysis. Kidney tissue lysates were extracted with $2 \%$ SDS buffer as previously ${ }^{28}$. Protein amounts were determined with the BCA kit from Thermo Scientific and equal amounts of protein were loaded in each lanes for SDS-polyacrylamide electrophoresis and then transferred to PVDF membrane. The blot was blocked with $5 \%$ milk for 1 hour at room temperature and then incubated with a specific primary antibody. The blot membrane was washed with TBST for 4 times and then incubated with horseradish peroxidase-conjugated secondary antibody, and signals were revealed using a chemiluminescence kit (Thermo Scientific).

Masson's trichrome staining of fibrosis. Renal fibrosis was shown by Masson's trichrome staining according to a standard protocol as described recently ${ }^{29}$. Briefly, the tissue was stained with hematoxylin, and then with ponceau red liquid dye acid complex, followed by incubation with phosphomolybdic acid solution. Finally, the tissue was directly stained with aniline blue liquid and acetic acid. The percentage of Masson staining positive area was determined in 10-20 microscopic fields for each sample, and ImageJ was used for quantitatively evaluation.

TUNEL assay. TUNEL assay was conducted by using the in situ Cell Death Detection kit from Roche as previously ${ }^{29,30}$. Paraffin-embedded tissue were deparaffinized and permeabilized with $0.1 \mathrm{M}$ sodium citrate, $\mathrm{pH} 6.0$ at $65^{\circ} \mathrm{C}$ for 1 hour. Then, the tissue section was incubated with the TUNEL reaction buffer for 1 hour at $37^{\circ} \mathrm{C}$ in a humidified chamber. The slides were mounted with ProLong Antifade Reagent. Positive staining was detected by Axioplan2 fluorescence microscope. In each tissue section, 10-20 fields were selected randomly to count positive staining cells.

Statiscal analysis. All values were expressed as mean \pm SD. Comparison between two groups was performed by paired Student t-test or unpaired student t-test. ANOVA were used to compare multiple groups. Data were analyzed with a Prism 6.0 software packet (San Diego, CA). Significance was considered by a $P$ value of $<0.05$.

\section{References}

1. Fox, C. et al. Inhibition of lysosomal protease cathepsin D reduces renal fibrosis in murine chronic kidney disease. Sci Rep 6, 20101, doi: 10.1038/srep20101 (2016).

2. Wouters, O. J., O’Donoghue, D. J., Ritchie, J., Kanavos, P. G. \& Narva, A. S. Early chronic kidney disease: diagnosis, management and models of care. Nat Rev Nephrol 11, 491-502, doi: 10.1038/nrneph.2015.85 (2015).

3. Wang, L. et al. Induction of secondary apoptosis, inflammation, and lung fibrosis after intratracheal instillation of apoptotic cells in rats. Am J Physiol Lung Cell Mol Physiol 290, L695-L702, doi: 10.1152/ajplung.00245.2005 (2006).

4. Elmore, S. Apoptosis: a review of programmed cell death. Toxicol Pathol 35, 495-516, doi: 10.1080/01926230701320337 (2007).

5. Wei, Q., Dong, G., Chen, J. K., Ramesh, G. \& Dong, Z. Bax and Bak have critical roles in ischemic acute kidney injury in global and proximal tubule-specific knockout mouse models. Kidney Int 84, 138-148, doi: 10.1038/ki.2013.68 (2013).

6. Degli Esposti, M. \& Dive, C. Mitochondrial membrane permeabilisation by Bax/Bak. Biochem Biophys Res Commun 304, 455-461 (2003).

7. Brooks, C. et al. Bak regulates mitochondrial morphology and pathology during apoptosis by interacting with mitofusins. Proc Natl Acad Sci USA 104, 11649-11654, doi: 10.1073/pnas.0703976104 (2007).

8. Chevalier, R. L., Forbes, M. S. \& Thornhill, B. A. Ureteral obstruction as a model of renal interstitial fibrosis and obstructive nephropathy. Kidney Int 75, 1145-1152, doi: 10.1038/ki.2009.86 (2009).

9. Goldner, J. A modification of the masson trichrome technique for routine laboratory purposes. Am J Pathol 14, 237-243 (1938).

10. Hironaka, K., Sakaida, I., Uchida, K. \& Okita, K. Correlation between stellate cell activation and serum fibrosis markers in cholinedeficient L-amino acid-defined diet-induced rat liver fibrosis. Digest Dis Sci 45, 1935-1943, doi: 10.1023/A:1005556328028 (2000).

11. Ina, K., Kitamura, H., Tatsukawa, S. \& Fujikura, Y. Significance of alpha-SMA in myofibroblasts emerging in renal tubulointerstitial fibrosis. Histol Histopathol 26, 855-866 (2011)

12. Bhatt, K. et al. MicroRNA-687 Induced by Hypoxia-Inducible Factor-1 Targets Phosphatase and Tensin Homolog in Renal IschemiaReperfusion Injury. J Am Soc Nephrol doi: 10.1681/ASN.2014050463 (2015).

13. Eizirik, D. L. \& Millard, I. [Chronicle of a death foretold: endoplasmic reticulum stress and beta cell apoptosis in diabetes]. Med Sci (Paris) 30, 496-499, doi: 10.1051/medsci/20143005007 (2014).

14. Pagliari, L. J. et al. The multidomain proapoptotic molecules Bax and Bak are directly activated by heat. Proc Natl Acad Sci USA 102, 17975-17980, doi: 10.1073/pnas.0506712102 (2005)

15. Scorrano, L. \& Korsmeyer, S. J. Mechanisms of cytochrome c release by proapoptotic BCL-2 family members. Biochem Biophys Res Commun 304, 437-444 (2003).

16. Jiang, X., Jiang, H., Shen, Z. R. \& Wang, X. D. Activation of mitochondrial protease OMA1 by Bax and Bak promotes cytochrome c release during apoptosis. Proc Natl Acad Sci USA 111, 14782-14787, doi: 10.1073/pnas.1417253111 (2014).

17. Antignani, A. \& Youle, R. J. How do Bax and Bak lead to permeabilization of the outer mitochondrial membrane? Curr Opin Cell Biol 18, 685-689, doi: 10.1016/j.ceb.2006.10.004 (2006).

18. Linkermann, A. et al. Regulated cell death in AKI. J Am Soc Nephrol 25, 2689-2701, doi: 10.1681/ASN.2014030262 (2014). 
19. Wei, Q., Dong, G., Franklin, J. \& Dong, Z. The pathological role of Bax in cisplatin nephrotoxicity. Kidney Int 72, 53-62, doi: 10.1038/sj.ki.5002256 (2007).

20. Johnson, A. \& DiPietro, L. A. Apoptosis and angiogenesis: an evolving mechanism for fibrosis. FASEB J 27, 3893-3901, doi: 10.1096/ f..12-214189 (2013).

21. Horowitz, J. C. et al. Constitutive activation of prosurvival signaling in alveolar mesenchymal cells isolated from patients with nonresolving acute respiratory distress syndrome. Am J Physiol Lung Cell Mol Physiol 290, L415-425, doi: 10.1152/ ajplung.00276.2005 (2006).

22. Wynes, M. W., Frankel, S. K. \& Riches, D. W. IL-4-induced macrophage-derived IGF-I protects myofibroblasts from apoptosis following growth factor withdrawal. J Leukoc Biol 76, 1019-1027, doi: 10.1189/jlb.0504288 (2004).

23. Havasi, A. \& Borkan, S. C. Apoptosis and acute kidney injury. Kidney Int 80, 29-40, doi: 10.1038/ki.2011.120 (2011).

24. van der Veer, W. M. et al. Time course of the angiogenic response during normotrophic and hypertrophic scar formation in humans. Wound Repair Regen 19, 292-301, doi: 10.1111/j.1524-475X.2011.00692.x (2011).

25. Jang, H. S. \& Padanilam, B. J. Simultaneous deletion of Bax and Bak is required to prevent apoptosis and interstitial fibrosis in obstructive nephropathy. Am J Physiol Renal Physiol 309, F540-550, doi: 10.1152/ajprenal.00170.2015 (2015).

26. Rankin, E. B., Tomaszewski, J. E. \& Haase, V. H. Renal cyst development in mice with conditional inactivation of the von HippelLindau tumor suppressor. Cancer Res 66, 2576-2583, doi: 10.1158/0008-5472.CAN-05-3241 (2006).

27. Takeuchi, O. et al. Essential role of BAX, BAK in B cell homeostasis and prevention of autoimmune disease. Proc Natl Acad Sci USA 102, 11272-11277, doi: 10.1073/pnas.0504783102 (2005).

28. Jiang, M. et al. Autophagy in proximal tubules protects against acute kidney injury. Kidney Int 82, 1271-1283, doi: 10.1038/ ki.2012.261 (2012).

29. Livingston, M. J. et al. Persistent activation of autophagy in kidney tubular cells promotes renal interstitial fibrosis during unilateral ureteral obstruction. Autophagy 12, 976-998, doi: 10.1080/15548627.2016.1166317 (2016).

30. Wei, Q. et al. MicroRNA-489 Induction by Hypoxia-Inducible Factor-1 Protects against Ischemic Kidney Injury. J Am Soc Nephrol 27, 2784-2796, doi: 10.1681/ASN.2015080870 (2016).

\section{Acknowledgements}

We thank Dr. Stanley Korsmeyer's laboratory at Dana-Farber Cancer Institute (Boston, MA) for providing Bax-floxed mouse line. We also thank Dr. Volker Haase at Vanderbilt University School of Medicine (Nashville, TE) for kindly providing PEPCK-Cre transgenic mice. This study was supported by the National key clinical specialist military construction project of China \{weiyiliao (2013) 147\}, Major Fundamental Research Program of Shanghai Committee of Science and Technology (12DJ1400300), Shanghai Top Priority Key Clinical Disciplines Construction Project, the National Institutes of Health and Department of Veterans Administration of USA. Zheng Dong is a senior Research Career Scientist of VA.

\section{Author Contributions}

S.M., C.M., and Z.D. designed the study, while S.M., L.L., Q.W. and J.H. performed experiments. S.M., Y.S., C.M., and Z.D. analyzed data and prepared manuscript for publication.

\section{Additional Information}

Competing Interests: The authors declare no competing financial interests.

How to cite this article: Mei, S. et al. Double knockout of Bax and Bak from kidney proximal tubules reduces unilateral urethral obstruction associated apoptosis and renal interstitial fibrosis. Sci. Rep. 7, 44892; doi: 10.1038/srep44892 (2017).

Publisher's note: Springer Nature remains neutral with regard to jurisdictional claims in published maps and institutional affiliations.

(c) (i) This work is licensed under a Creative Commons Attribution 4.0 International License. The images (c) or other third party material in this article are included in the article's Creative Commons license, unless indicated otherwise in the credit line; if the material is not included under the Creative Commons license, users will need to obtain permission from the license holder to reproduce the material. To view a copy of this license, visit http://creativecommons.org/licenses/by/4.0/

(c) The Author(s) 2017 